\title{
DYNAMICS OF HOT ELECTRONS IN ZnSe-ZnTe DOUBLE BARRIER HETEROSTRUCTURES
}

\author{
P. Bala \\ Institute of Physics, N. Copernicus University, Grudziądzka 5, 87-100 Toruń, Poland
}

In this paper we perform a detailed study of the transport of hot electrons in the double barrier heterostructures with the presence of a collector barrier. This system is considered as a double barrier resonant tunneling device. The electron is described by time-dependent Schrödinger equation, which allows us to study detailed dynamics of the carriers. The influence of an energy step in the collector area of the device on the tunneling probability is investigated. The significant role of dissipation due to electron-phonon interactions is presented.

PACS numbers: 03.65.-w, 73.40.Gk, 73.61.-r

Resonant tunneling has been the topic of great interest in recent years and has been intensively studied using simple electronic devices such as double barrier resonant tunneling heterostructures (DBRT). The DBRT structures are also used as a source of the hot electrons injected to the rest of the device and can be implemented into electronic devices such as resonant-tunneling hot electron transistors (RIIETs) [1,2]. The growing number of practical applications increases interest in investigation of the electron dynamics in such devices [3, 4].

The improvement of the technology of device fabrication and measurement techniques allows nowadays for investigation of different aspects of carrier transport in a wide range of the double barrier structures. Available experimental data show well-interpreted resonant tunneling peaks in $I-V$ characteristics. However, additional peaks can also be observed $[5,6]$. The interpretation of most of the observed data is well established based on resonant tunneling as the most important transport process, but the exact nature of the additional peaks is not clear. One of the possible explanation is a sequential energy loss of electron's kinetic energy due to the phonon emission [5]. The sequential energy loss is possible while the ballistic electron interacts strongly with the phonon bath. Another approach stresses the role of quantum interference effects in the collector region of device [6]. This approach stresses the role of the additional energy barriers present in the system at the collector side of the device. Because of the difficulty in obtaining direct experimental evidence which can confirm or eliminate presented explanations, a consistent theoretical treatment can provide additional information.

In the relaxation process, as well as in interference of the electron wave function on the potential step, the essential role is played by a relatively high 
electron energy which must be dissipated to the environment. One of the important dissipation processes is electron-phonon interaction which allows for the transfer of electron excess energy to crystal.

The standard description of the electron in heterostructures is based on the stationary approach and neglects dynamical effects. This method succeeded to explain a wide range of effects joined to the tunneling and to the resonant tunneling, although the plane wave approach to the electron in the semiconductor cannot be applied in more advanced situations. Especially, the usage of this approach to the hot electrons with high kinetic energy in the double barrier heterostructures and other devices is at least questionable.

The aim of this paper is to investigate the influence of the energy step as well as electron-phonon interactions in the collector area of the double barrier resonant tunneling device on the tunneling probability. A standard description of a system interacting with crystal environment is based on a phonon bath as a set of simple harmonic oscillators $[7,8]$. The phonon bath is then treated in the stationary approach and no information about tunneling dynamics is obtained.

In this paper we introduce, to the time-dependent description of tunneling phenomena [9-11] interaction of the carriers with the phonons. This allows us to obtain a proper analysis of phonon-electron interactions in the collector region of device. The interaction of the carriers with the phonons is described, in the first approximation, as the additional periodic, time-dependent potential added to the device band structure in the collector area. The potential responsible for electron-phonon coupling has a form of the traveling wave with the frequency $\omega_{\mathrm{p}}$ and the wave vector $k_{\mathrm{p}}$

$$
V_{\mathrm{p}}(x, t)=\mathcal{E}_{\mathrm{p}} \cos \left(\omega_{\mathrm{p}} t-k_{\mathrm{p}} x\right), \quad x>d_{\mathrm{c}} .
$$

The frequency of the wave can be associated with the measured phonon energy $E_{\mathrm{ph}}$ through the energy-frequency dependence: $E_{\mathrm{ph}}=\hbar \omega_{\mathrm{p}}$. In the same way the wave vector corresponds to the characteristic dimension in the system, $2 \pi / k_{\mathrm{p}}$.

The double barrier resonant tunneling structure is formed by an $n$-type $\mathrm{ZnSe}$ layer of a width $d_{\mathrm{W}}=47.6 \AA$ (quantum well) surrounded by $p$-type $\mathrm{ZnTe}$ layers of a width $d_{\mathrm{B}}=26.4 \AA$ (barrier region). The height of the barrier is $V_{0}=0.59 \mathrm{eV}$ which results in the presence of one discrete energy level in the well $\left(E_{0}=0.38 \mathrm{eV}\right)$. The structure is placed between the wide $n$-type ZnSe layers (with a length greater than $3500 \AA$ ). In the collector region the additional region of a width $d_{c}=1000 \AA$ is introduced to form space buffer just after the double barrier resonant tunneling device (Fig. 1). The rest of the collector area for $x>d_{\mathrm{c}}$ forms either the constant potential barrier $V_{\mathrm{p}}$ or is modified by the electron-phonon interaction potential $V_{\mathrm{p}}(x, t)$.

The system is investigated under the constant voltage $V$ applied along the growth direction. Linear potential changes are assumed only in the barrier regions [11]. The electron dynamics is obtained by solving, within effective mass approximation [12], the time-dependent Schrödinger equation

$$
\mathrm{i} \hbar \frac{\partial \Psi(x, t)}{\partial t}=\left(-\frac{\hbar^{2}}{2 m^{*}} \frac{\partial^{2}}{\partial x^{2}}+V_{\mathrm{B}}(x)+V_{\mathrm{p}}(x, t)\right) \Psi(x, t)
$$

where $m^{*}$ is the electron effective mass and depends on the layer type $\left(m_{\mathrm{ZnSe}}^{*}=\right.$ 
$\left.0.16 m_{\mathrm{e}}, m_{\mathrm{ZnTe}}^{*}=0.32 m_{\mathrm{e}}\right)$, and $V_{\mathrm{B}}(x)$ denotes the static double barrier potential with the applied external voltage applied to the structure. The time-dependent Schrödinger equation (2) is solved using discrete grid methods as it was reported previously $[13,14]$. The initial wave function is of the Gaussian shape, and its parameters, time step and the grid size are the same as in the previous works $[10,15]$.

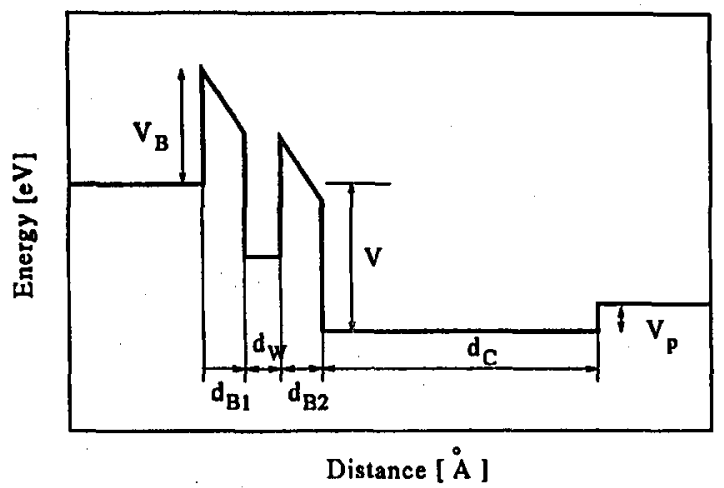

Fig. 1. Energy diagram of the investigated structure.

The tunneling probability calculated with the absence of the additional potentials exhibits a single resonant peak at the applied voltage $V_{\text {peak }}=0.77 \mathrm{~V}$.

The presence of the energy step in the collector area of the double barrier resonant tunneling structure does not change the tunneling probability significantly (Fig. 2a). The height of the barrier was changed from $0.01 \mathrm{eV}$ up to the $0.3 \mathrm{eV}$ (half of the barrier height). In the whole range there is no significant change in the tunneling. As it is presented in Fig. 2b the additional barrier in the collector region changes slightly the charge trapped in a region just behind the DBRT device. In the absence of the additional energy barrier the total charge accumulated in the $1000 \AA$ buffer reaches maximum at time 0.4 ps and then decreases exponentially as the transmitted part of the wave packet moves forward to the collector. The time dependence of the charge, presented in Fig. $2 \mathrm{~b}$ for the applied voltage $V=0.74 \mathrm{~V}$, shows also small oscillations in the decay caused by the difference of incident electron energy and energy of the discrete level in the quantum well [9].

While the additional energy step is present, the charge accumulated in the buffer region increases and then decreases exponentially. For the time longer than $0.7 \mathrm{ps}$ small differences in the charge decay are observed. The presence of the energy step causes accumulation of the charge in the wide well formed by the second barrier of the DBRT device and energy step in the collector area. The total charge accumulated in this area depends on the height of the barriers, especially on $V_{p}$ but it is very small and practically has no influence on the tunneling characteristics even for relatively high potential energy steps $\left(V_{\mathrm{p}}=\frac{1}{2} V_{0}\right)$. The tunneling characteristics are almost identical and small differences are seen only in the first derivative of the tunneling probability. 
Since the step barrier in the collector area does not influence significantly tunneling probability, the eiectron-phonon coupling was introduced in the collector area. The applied frequency corresponds to the $\mathrm{ZnSe}$ LO phonon energy of $31 \mathrm{meV}$ [16], and wave vector $k_{\mathrm{p}}$ corresponds to $30 \AA$. With an increase in the electron-phonon coupling the significant changes are observed in the tunneling probability. The decrease in the tunneling probability is observed at a voltage of $0.7 \mathrm{~V}$ even for a small coupling (Fig. 3a). A further increase in $E_{\mathrm{p}}$, performed to emphasise the influence of the electron-phonon coupling, causes the overall decrease in the tunneling probability. Additional peaks are also observed together with a complicated structure of the tunneling probability. Peaks corresponding to
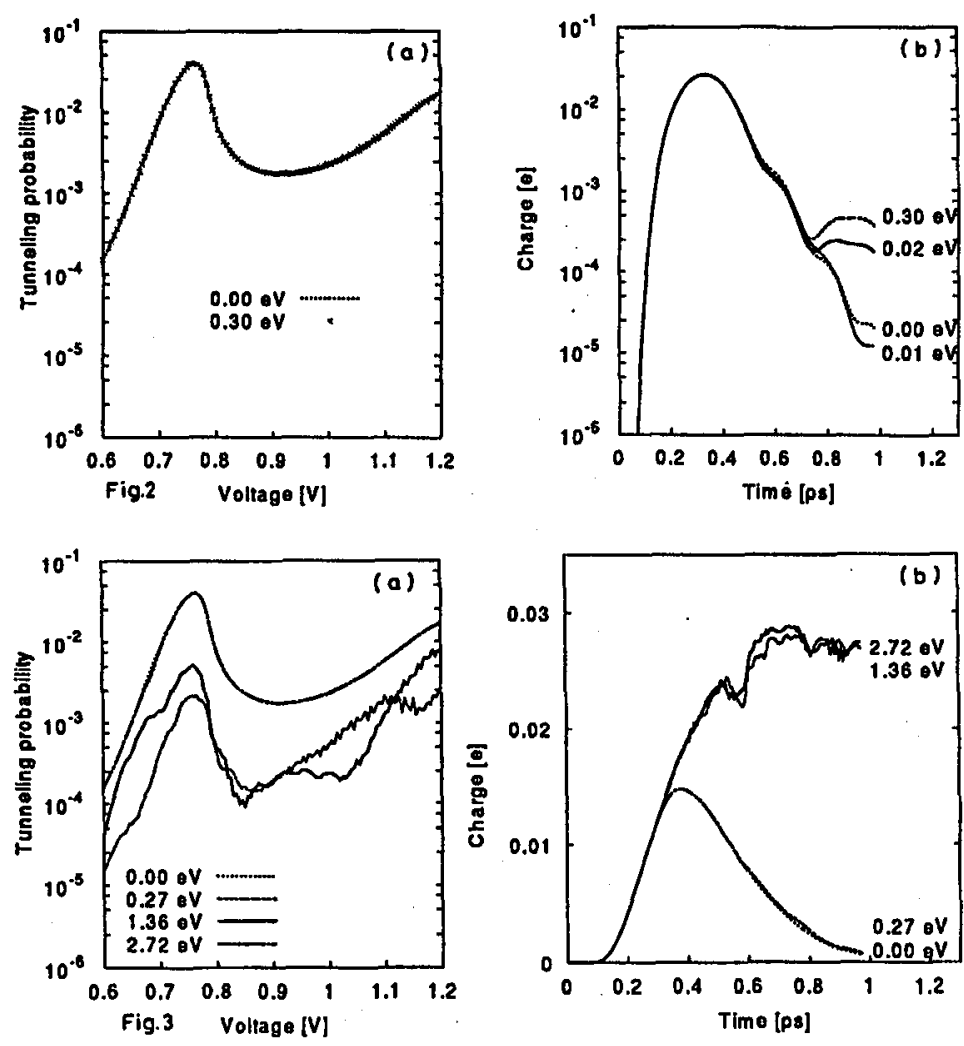

Fig. 2. Tunneling probability (a) for the double barrier $\mathrm{ZnTe} / \mathrm{ZnSe} / \mathrm{ZnTe}$ structure without (line) and with the potential step $V_{p}=0.30 \mathrm{eV}$ in the collector area and total charge (b) accumulated in the area between DBRT structure and potential step for different step heights $V_{\mathrm{p}}$, for the applied voltage $V=0.74 \mathrm{~V}$.

Fig. 3 Tunneling probability (a) for the double barrier $\mathrm{ZnTe} / \mathrm{ZnSe} / \mathrm{ZnTe}$ structure without, $\mathcal{E}_{\mathrm{p}}=0.0 \mathrm{eV}$, and with the electron-phonon interactions in the collector area and total charge (b) accumulated in the area between DBRT structure and potential step for different electron-phonon couplings $\mathcal{E}_{\mathrm{p}}$ at applied voltage $V=0.78 \mathrm{~V}$. 
the tunneling through quasi levels separated by the phonon fundamental energy $\hbar \omega_{\mathrm{p}}$ are not observed. Additional peaks seen at much larger energy distances from the original tunneling peak can be associated with multiphonon processes, or with more complicated resonances. The time-dependent approach allows for detailed investigation of the suppression of the tunneling probability. As presented in Fig. 3b the decrease in the tunneling probability is caused by charge trapping in the region just behind the double barrier resonant tunneling structure. The electron-phonon potential reduces significantly a part of the wave function which reaches the collector. Therefore, a charge which tunnels through the DBRT structure is accumulated in the buffer region. The charge build-up prevents further tunneling and tunneling probability is reduced. In the presented model the charge build-up does not influence the valence band structure of the device. In general charge accumulation behind a barrier can modify a barrier height and further reduce tunneling probability.

In conclusion, the detailed time-dependent picture of the phonon assisted resonant tunneling phenomena in the double barrier structure is presented. The presented data show significant influence of the electron-phonon scattering on the tunneling process. The interaction reduces the tunneling probability and leads to the additional structure of the tunneling probability characteristics. The very limited influence of the additional barrier in the collector area on the hot-electron dynamics is shown. The obtained results allow for understanding the tunneling process and for better interpretation of the experimental data.

This work is supported in part by the Committee for Scientific Research under grant No. P03B 156 10. The calculations were partially performed in the Interdisciplinary Centre for Mathematical and Computational Modelling at Warsaw University.

\section{References}

[1] N. Yokoyama, K. Imamura, M. Takatsu, T. Mori, T. Adachihara, Y. Sugiyama, Y. Sakuma, A. Tackeuchi, S. Muto, Philos. Trans. R. Soc. Lond. A, Math. Phys. Eng. Sci. 354, 2399 (1996).

[2] N. Yokoyama, S. Muto, K. Imamura, M. Takatsu, T. Mori, Y. Sugiyama, Y. Sakuma, H. Nakao, T. Adachihara, Solid-State Electron. 40, 505 (1996).

[3] M.Y. Frankel, G.L. Belenky, S. Luryi, T.F. Carruthers, M.L. Dennis, A.Y. Cho, R.A. Hamm, D.L. Sivco, J. Appl. Phys. 79, 3312 (1996).

[4] K.-M. Hung, T.-N. Fang, C.-J. Chen, C. Chang, G.Y. Wu, Solid State Commun. 92,625 (1994).

[5] M.V. Petrov, S.R. Parihar, S.A. Lyon, Phys. Rev. B, Condens. Matter 54, 13868 (1996).

[6] T. Figielski, T. Wosiński, S.A. Vitusevich, A.E. Belyaev, A. Mąkosa, W. Dobrowolski, Semicond. Sci. Technol. 12, 86 (1997).

[7] J.A. Sanchez-Gil, Phys. Rev. B, Condens. Matter 53, 10317 (1996).

[8] J.A. Stovneng, E.H. Hauge, P. Lipavski, V. Spicka, Phys. Rev. B 44, 13595 (1991).

[9] P. Bała, Electron Technology 29, 63 (1996).

[10] P. Bała, R. Ryszewski, W. Bała, Acta Phys. Pol. A 84, 685 (1993). 
[11] P. Bała, W. Bała, Appl. Phys. A, Solids Surf. 60, 293 (1995).

[12] R. Tsu, L. Esaki, Appl. Phys. Lett. 2, 562 (1973).

[13] P. Bała, J.S. Kwiatkowski, W. Bała, Acta Phys. Pol. A 82, 649 (1992).

[14] H. Tel-Ezer, R. Kosloff, J. Chem. Phys. 81, 3967 (1984).

[15] P. Bała, R. Ryszewski, W. Bała, Acta Phys. Pol. A 87, 341 (1995).

[16] L. Samuel, Y. Brada, A. Burger, M. Roth, Phys. Rev. B, Condens. Matter 36, 1168 (1987). 